\title{
Positronium Trapping in Porous Solids: Means and Limitations for Structural Studies
}

\author{
Zs. $\operatorname{Kajcsos}^{a}$, L. Liszkay ${ }^{a}$, G. Duplâtre ${ }^{b}$, L. $\operatorname{VArgA}^{a}$, \\ L. LOHONYAi ${ }^{a}$, F. PÁSzti ${ }^{a}$, E. SzIlágyi ${ }^{a}, \mathrm{~K}$. LÁzÁR ${ }^{c}$, \\ E. Kótai ${ }^{a}$, G. PÁl-Borbély ${ }^{d}$, H.K. Beyer ${ }^{d}$, P. CAullet ${ }^{e}$, \\ J. Patarin ${ }^{e}$, M.E. Azenha ${ }^{f}$, P.M. Gordo ${ }^{g}$, C. Lopes Gil ${ }^{g}$, \\ A.P. DE Lima ${ }^{g}$ AND M.F. FERREIRA MARQUES ${ }^{h}$
}

${ }^{a}$ KFKI Res. Inst. for Part. Nucl. Physics, P.O.B. 49, 1525 Budapest 114, Hungary

${ }^{b}$ Lab. Chim. Nucléaire, IReS, IN2P3/CNRS, B.P. 28, 67037 Strasbourg Cedex 2, France

${ }^{c}$ Inst. of Isotope and Surface Chemistry, P.O.B. 77, 1525 Budapest, Hungary

${ }^{d}$ Centr. Res. Inst. for Chemistry, P.O.B. 19, 1525 Budapest, Hungary

${ }^{e}$ Lab. Mat. Minéraux, URA CNRS 4283, rue A. Werner, 68093 Mulhouse Cedex, France

${ }^{f}$ Dept. of Chemistry, University of Coimbra, Coimbra 3004-535, Portugal

${ }^{g}$ Dept. of Physics, University of Coimbra, Coimbra 3004-516, Portugal

${ }^{h}$ Instituto Superior de Engenharia, Coimbra 3004-535, Portugal

Positron annihilation techniques are used for the structural investigation of solids but the interpretation of results in grainy and porous media is still unclear. A unique picture can be obtained assuming that the dominant process is Ps trapping in competing "extended free volume" sites. In samples with a large amount of free volumes near-saturation Ps trapping will rule the lifetime pattern, and very long lifetimes of over $100 \mathrm{~ns}$ might arise from $o$-Ps trapped in mesopores. It is shown that lifetime parameters must be corrected for the $3 \gamma / 2 \gamma$ counting efficiency ratio. The results demonstrate the high sensitivity of Ps to mesopores in zeolites but also that Ps-trapping poses limitations on the applicability of lifetime to structural investigation in porous systems. The evolution of the lifetime spectra upon changes in the sample and measuring conditions should be considered in a complex way, observing not only changes in some selected components but in the whole lifetime pattern simultaneously.

PACS numbers: 36.10.Dr, 78.70.Bj, 61.43.Gt 


\section{Introduction}

Positron annihilation techniques (PAT) became routine-like methods for the structural investigation of solids but the interpretation of results gained by positron lifetime spectroscopy (LT) in grainy and porous media was and still is ambiguous. Generally, at least one long lifetime component attributed to ortho-positronium (o-Ps) was observed [1, 2], but recent LT measurements have revealed the presence of a higher number of long components, all connected with various $o$-Ps states formed $[3,4]$. In solids, the interacting $\mathrm{e}^{+} / \mathrm{e}^{-}$pair can develop into Ps at the surface of open sites, o-Ps becoming rapidly trapped thereafter in open spaces inside the solid matrix and decays therein. Inhomogeneous regions and grain boundaries may also act as Ps traps. Whereas the intrinsic vacuum decay of o-Ps (with 142 ns lifetime) results in the emission of three $\gamma$ rays, its extrinsic decay in matter is governed by the overlap of the positron wave function with that of electrons of the surrounding medium, and this fast "pick-off" process results in a decay with essentially $2 \gamma$-emission with much shorter lifetimes. Due to this shortening, the lifetime of localized $o$-Ps is sensitive to the size of the trap: the larger the free volume, the longer the lifetime. The observation of $o$-Ps states allows an indirect characterization of solid materials where free volume patterns are of high practical importance (nano- and mesoporous materials, micrograin powders, polymers, etc.). The most extensively used quantitative expression $[5,6]$ correlates the $o$-Ps lifetime $\tau$ with the radius of the (presumably, spherical) void, $R$ :

$$
1 / \tau=2\left[1-R / R_{0}+(1 / 2 \pi) \sin \left(2 \pi R / R_{0}\right)\right]
$$

with $\tau$ in ns and $R_{0}=R+0.1656(\mathrm{~nm})$. This equation has also been modified $[7,8]$ to take other shapes of the voids than spherical into account, providing a unique method to determine free volume sizes in a range of about $0.1-10 \mathrm{~nm}$. These equations have been applied successfully for samples with intrinsic or extrinsic defects including mesopores, or free volumes in polymers. It was an obvious step from the beginning of the discovery of long-lived o-Ps components in microporous solids that highly porous samples with a substantial amount of free volume in the bulk could serve for the proof of this model and for the extension of validity. Among the possible materials, zeolites represent a promising field of investigation with direct technological importance to obtain information regarding both the probe (Ps) and the solid matrix. Zeolites contain a variety of well defined open spaces, a net of channels, cages isolated from the channels. Depending on the preparation method, defected sites and mesopores can also form, whose size and arrangement depend on the specific synthesis, e.g., on the templates and on the proportion of metal ions substituting the $\mathrm{Si}$ ions. This variety of non-occupied volumes can give rise to a number of localized $o$-Ps states. The difficulty of resolving the LT decay spectra into a large number of components, however, is also accompanied with several factors which may also introduce undesired artifacts strongly influencing the data. For example, the synthesis procedure can result in a variable amount 
and size of defects and pores in nominally identical samples and differences in water content of the zeolites [9] may play an important role, too.

As the size of the trapping sites is mirrored in the respective lifetime value, it is expected that the amount of the specific free volume would be reflected by the $o$-Ps intensity and quantitative information could be obtained on the concentration of the $o$-Ps trapping centres as is the case with positron trapping experiments in solid state physics studies. This expectation, however, raises the question of the relevance of the measured o-Ps intensity, opposed to its true, physical intensity. The smaller the trapping site accommodating the $o$-Ps, the higher the $2 \gamma$-decay fraction will be, and vice versa. Obviously, although usually overlooked, the overall counting efficiency may drastically differ for those two decay modes $[10,11]$ and the specific geometry and setting of the LT apparatus can influence the measured relative amounts of the $o$-Ps states resulting in falsified relative intensities of the various positron and Ps states. The knowledge of the true LT intensities requires the knowledge of the ratio of the $3 \gamma / 2 \gamma$ counting efficiencies, $\rho$ [10-12]. To this end, various experimental procedures have been proposed, transforming the long-lived, $3 \gamma$-emitting o-Ps states into short-lived ones, e.g., by applying either chemical quenching [11] or an external magnetic field [12]. We have recognized that those methods have various drawbacks and a stand-alone method with easier means to control the $3 \gamma / 2 \gamma$ ratio was also aimed at: we have exploited our earlier findings $[13-15]$ showing that with gas filling the long-lived $o$-Ps intensities may gradually disappear as a function of temperature $(T)$.

Here we report on some further results on the extensive series of studies carried out with the aim of understanding the formation and fate of Ps in solids better and with that of characterizing some properties of zeolites. The fate of Ps was studied in silicalite-1 [4], one of the less complex zeolite species consisting purely of $\mathrm{SiO}_{2}$, because of its "simplicity" thought to be appropriate to expose interactions between Ps and the basic constituents of the zeolites. It was also our aim to investigate the validity of the $o$-Ps trapping assumption.

\section{Experimental}

As the selected research goal requests the observation of $3 \gamma$-decaying long-lived o-Ps states, PAT was oriented to the methods best suited for this task. Apart from conventional LT and Doppler broadening (DB) measurements, the full energy distribution (FED) of the annihilation radiation was also recorded. Comparing LT with DB and FED results may help to determine the true intensities of the long-lived $o$-Ps components, whose recording is affected by the energy (and solid angle) dependent efficiency $\rho$ of recording $2 \gamma$ - and $3 \gamma$-events, respectively. The LT measurements were primarily carried out in Budapest and in Strasbourg $[4,11]$, employing high quality spectrometers (with 250 ps and 350 ps FWHM, respectively) set for high efficiency recording of $3 \gamma$-events, i.e., the energy windows 
of the detectors were set to $50 \%$ for the start and to $80 \%$ for the stop signals, as scaled to the upper discrimination level. LT spectra with integral counts of $5 \times 10^{6}-4 \times 10^{7}$ were collected in a time range of $1-2.5 \mu \mathrm{s}$. The FED spectra were taken in Budapest by a Canberra HPGe detector (25\% efficiency, $1.1 \mathrm{keV}$ resolution at $0.5 \mathrm{MeV}$ ) into a Microfast $16 \mathrm{k} \mathrm{PC}$ analyzer in the $40-1350 \mathrm{keV}$ range (monitoring the number of positrons by the $1.28 \mathrm{MeV}$ photo-peak), allowing so to extract the conventional DB parameters as well from the same recording. Test and control measurements were also repeated in Coimbra with spectrometers of similar specifications, proving good data reproducibility. To gain information on the sensitivity of PAT to sample differences, three production series of silicalite-1 (structural type MFI, with 96 individual $\mathrm{SiO}_{2}$ groups per $5.351 \mathrm{~nm}^{3}$ volume unit cell, specific mass: $1.79 \mathrm{~g} \mathrm{~cm}^{-3}$ ) were selected for sample collections $A, B$, and $C$. Samples $A$ and $B$, synthesized in fluorated media consisted of uniform dimension high quality crystallites $\left(30 \times 8 \times 6 \mu \mathrm{m}^{3}\right.$ and $150 \times 30 \times 20 \mu \mathrm{m}^{3}$, respectively), while specimens $C$ with crystallites of $15 \times 5 \times 5 \mu \mathrm{m}^{3}$ were prepared in the presence of $\mathrm{OH}^{-}$ions, known to result in samples with more lattice defects [16]. The specimens were calcined for 4 hours at $823 \mathrm{~K}$ in air, adsorbed water was removed by heating them for $48 \mathrm{~h}$ at $623 \mathrm{~K}$ in vacuum. Sample characterization has been performed in Mulhouse by X-ray powder diffraction, solid state NMR, chemical analysis, whereas nitrogen adsorption measurements gave information on the zeolite pore volume amount. The MFI matrix features a network of straight and zigzag channels of $0.55 \mathrm{~nm}$ average diameter. Sample $B$ was studied as powder, while the others were pressed by $0.3 \mathrm{GPa}$ into $8 \mathrm{~mm}$ diameter, $1.5 \mathrm{~mm}$ thick pills. Samples $A$ and $C$ consisted of two pressed zeolite disks embodying a ${ }^{22} \mathrm{Na}$ source of about $1 \mathrm{MBq}$ sealed between thin kapton or Ni foils, necessitating only a single-component source correction of several percents. The sample-source compound was placed in a glass ampoule, either evacuated or filled with $\mathrm{N}_{2}$ of spectroscopic purity at pressures in the range of $0.25-1$ atm and sealed subsequently. Sample $B$ was packed in a small aluminium container, embedding the carrier-free $0.5 \mathrm{MBq}$ ${ }^{22} \mathrm{Na}$ droplet, dried up directly on the lower powder cushion, so that there was no need for source correction. The samples were studied as a function of temperature $(T)$ in the $83-294 \mathrm{~K}$ range [13-15].

\section{Results and discussion}

As was published earlier $[12,13,15,17]$ and mentioned here previously, LT spectra might substantially be distorted by differences in the recording efficiency for $2 \gamma$ - and $3 \gamma$-annihilation events, respectively. The setting of the energy window on the stop detector line strongly influences the relative intensity of the components denoting $3 \gamma$-annihilation. The correction factor $\rho$ shows an overweighting of the $3 \gamma$-annihilation events with respect to the same number of $2 \gamma$-annihilation events. Assuming equal detection efficiency and no solid angle dependence, a naïve 


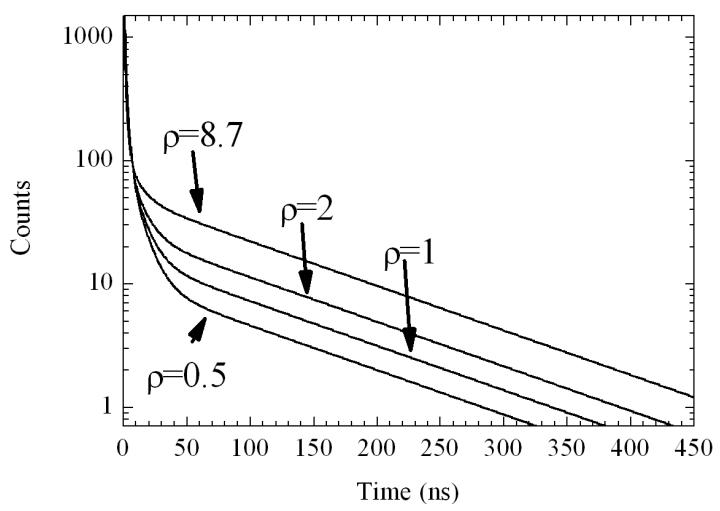

Fig. 1. Positron lifetime spectrum measured with instruments of various $\rho$ value (simulation, long time range).

expectation would be $\rho=1.5$, but for various experimental conditions values between 1-8.7 have been published [10]. Assuming a LT distribution containing four lifetimes with $0.4 \mathrm{~ns}, 2 \mathrm{~ns}, 10 \mathrm{~ns}$, and $120 \mathrm{~ns}$ and physical intensities of 50-2010-20 percents, LT spectra simulated assuming various $\rho$ values are presented in Fig. 1. The lifetime values do not change upon $\rho$ but the intensities are strongly influenced. To determine the real intensities, one has to scale the $2 \gamma$ - and $3 \gamma$ annihilation events by the $\rho$ values back to their real physical fraction. For the determination of $\rho$ we exploited our earlier results showing that by cooling the long-lived $o$-Ps components could be influenced, up to a complete disappearance of the long contribution at low temperatures [4] with a slope depending on the amount of the gas present. This result shows that gas capillary condensation into the zeolite eliminates the long-lived components of 40-130 ns [4, 14] and only lifetimes of several ns subsist; the intensity of the longest-lived components diminishes whereas the shorter-lived components become dominant (examples are shown in Fig. 2), proving that not the formation of Ps is inhibited but rather the $o$-Ps lifetime is shortened when entering the liquid-like gas condensate $[4,11]$. The intensities shown here were not corrected for $\rho$, they were exploited for extracting the actual values of $\rho$. The normalized total integrals of the LT spectra were compared, the difference in the integrals is a function of $\rho$ alone, as it will be elaborated in more detail in a dedicated work in progress [18].

LT spectra were analyzed into four and five components with intensities $I_{i}$ and lifetimes $\tau_{i}$ by a fitting program based on the MINUIT program package; this large number of components has been shown to be justified previously $[12,14,18]$. Spectrum analysis into less components resulted in inferior fits. Some indicative LT parameters for the different samples are given in Table, intensities before and after correction with the respective $\rho$ values. An overall good agreement for the powder and pressed pill samples is observable for the longest component, whereas the intermediate ones exhibit higher fractions at the expense of the short one. The 

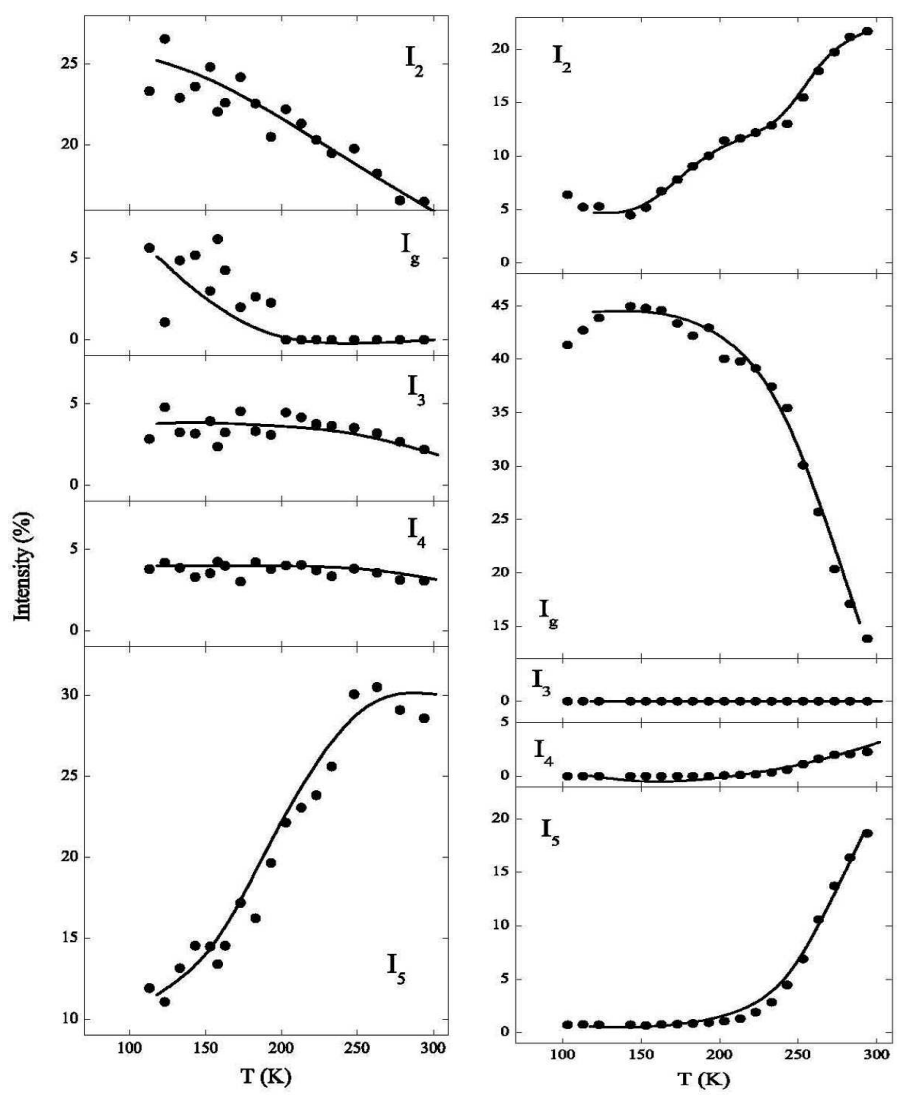

Fig. 2. Temperature dependent evolution of various lifetime components for sample $A$ from the five-component LT evaluation (left side: 0.25 atm $\mathrm{N}_{2}$ pressure; right side: 1 atm $\mathrm{N}_{2}$ pressure). The intensity values $I_{2}, I_{\mathrm{g}}, I_{3}, I_{4}$, and $I_{5}$ are shown from up to down, $I_{\mathrm{g}}$ is the component which represents the gas filling and appears only at low temperatures at the expense of the previous long components.

various components may be categorized as follows: $\tau_{1}$ with $0.2-0.5 \mathrm{~ns}$ combining the $p$-Ps and $\mathrm{e}^{+}$annihilations, $\tau_{2}$ with 1-4 ns for $o$-Ps probably trapped in small voids of the lattice, $\tau_{3}$ with $10-20 \mathrm{~ns}$ for $o$-Ps decaying in internal channels of the zeolites and $\tau_{4}$ with 50-90 ns and $\tau_{5}$ with 100-140 ns for $o$-Ps decaying outside of the crystallites, in the intergrain space or in very extended free volumes. Due to the high time/channel setting used, the absolute values of the shortest lifetime $\tau_{1}$ are less reliable than the longer ones and will not be considered here. The longer- but still comparatively short-components might be identified with intrinsic structural free volumes present in silicalite-1; the trapping of $o$-Ps in a potential well with decay lifetimes of about 10-30 ns yields sizes of the localization sites that are very close to the ones expected on crystallographic grounds [19]. However, there is no such conformity for lifetimes of about $100 \mathrm{~ns}$, which finding either points to flaws 
TABLE

Lifetimes and relative intensities for the three basic samples, as measured and corrected with the respective $\rho$ values determined comparing room temperature and low temperature LT spectra [14]: lifetimes are not influenced by the correction. (The errors on the intensities are \pm 0.2 abs.\%.)

\begin{tabular}{c|c|c|c|c|c|c|c|c|c}
\hline \hline Sample & $\tau_{2}[\mathrm{~ns}]$ & $\tau_{3}[\mathrm{~ns}]$ & $\tau_{4}[\mathrm{~ns}]$ & $\tau_{5}[\mathrm{~ns}]$ & $I_{2}[\%]$ & $I_{3}[\%]$ & $I_{4}[\%]$ & $I_{5}[\%]$ & $\rho$ \\
\hline$A$ (meas.) & 2.05 & 5.60 & 35.1 & 135.4 & 24.1 & 2.6 & 2.5 & 28.1 & 1.78 \\
& \pm 0.03 & \pm 0.2 & \pm 1.9 & \pm 1.4 & & & & & \\
$A$ (corr.) & & & & & 27.2 & 2.9 & 2.4 & 18.5 & \\
$B$ (meas.) & 1.32 & 5.58 & 37.7 & 124.5 & 20 & 2.0 & 3.3 & 27.4 & 1.64 \\
& \pm 0.02 & \pm 0.07 & \pm 2.3 & \pm 1 & & & & & \\
$B$ (corr.) & & & & & 22 & 2.2 & 3.1 & 18.0 & \\
$C$ (meas.) & 2.24 & 7.5 & 33.2 & 123.3 & 5.5 & 10.8 & 8.6 & 21.2 & 1.30 \\
& \pm 0.09 & \pm 0.2 & \pm 1.1 & \pm 1.6 & & & & & \\
$C$ (corr.) & & & & & 5.8 & 11.0 & 8.4 & 17.4 &
\end{tabular}

in the model as regards the expansion of the Ps wave function into the lattice bulk, or it must be assumed that the sample contains more extended free volume sites. The $o$-Ps trapping model yields a radius of about $10-12 \mathrm{~nm}$ for them, values typical of mesopores and also evidenced by the low temperature $\mathrm{N}_{2}$ adsorption measurements for the defected sample. That also the "undefected" samples exhibit this component implies that the trapping efficiency of those extended trapping sites is so high that even if their amount is still not visible by other methods they suffice to trap o-Ps during pre-thermalization diffusion. For the evaluation of DB and FED data, the $L / R$ values (left/right plateau regions of the $511 \mathrm{keV}$ peak, [4]) enable the scaling of the fraction $f_{3 \gamma}$ of long-lived $o$-Ps, decaying by $3 \gamma$-emission. Comparing LT and FED data [14], good agreement was found between $f_{3 \gamma}$ and the LT intensity values corrected for $\rho$.

\section{Conclusions}

The porous media still present a very challenging terraine for positron annihilation studies. The present results lead to the conclusion that the LT pattern in zeolites might after all be interpreted by the "free volume" trapping of $o$-Ps, accepting the assumption that in addition to the structural free volumes "out of structure" mesopores can also play a dominant role. The simultaneous presence of short and long lifetime components extending well over three orders of magnitude in time makes the LT measurements difficult. At the same time, the intensities of the lifetime components may also differ over one order of magnitude. In addition to the need of high statistics enabling the decomposition of LT spectra into several components with acceptable reproducibility and significance, appropriate computer codes must also be developed for that. It is demonstrated that without 
correcting the detection efficiency differences for the $2 \gamma$ - and $3 \gamma$-decay modes the lifetime values could be very significantly false. A combination of various PAT methods may help to carry out the proper physical scaling of the competing annihilation modes. To minimize data interpretation ambiguities, special attention has to be paid to controlling the sample preparation and the external conditions of the positron annihilation measurements. The LT spectra should be considered in a complex way as an organic whole, and not only selected components should be observed and scrutinized. The evolution of the LT spectra as a function of e.g. some external parameters requires also the observation of the transitions between various annihilation modes. Only this kind of mapping of changes enables distinctions between various interactions leading to virtually identical "end symptoms" (e.g., Ps-inhibition or o-Ps conversion would both result in the decrease in the intensity of the long lifetime components) to be differentiated only by looking at the whole picture.

The trapping of $o$-Ps - eventually at near-saturation level - shadows sensitivity to structural features, showing great similarity to the behaviour of positrons in amorphous glasses where a saturation level trapping masks the possible influence of structural changes on the annihilation pattern. The high level of trapping, however, may allow to study the features of the internal walls by changes of the respective lifetimes upon e.g. ionic changes influencing the dielectric constant of the wall. This aspect of PAT applications might eventually be exploited in the $\mathrm{SiO}_{2}$-using semiconductor technology as well, together with ion beam technique applications exhibiting high sensitivity to some specific low-concentration impurities. Slow positron measurements should also be performed to clarify some questions mentioned before.

As a consequence of the concentrated efforts in the last decade, it became clear that there are still theoretical questions but also experimental difficulties that were not dealt with thoroughly enough. The influence of the incomplete thermalization, delayed formation of Ps, the diffusion of o-Ps before getting trapped, a contribution of long lifetimes instead of discrete values, the possibility of transitions between various trapped states and that of the detrapping should be considered in detail, a very complicated and challenging task. Work along these lines to put together the pieces of mosaic to a whole picture - is in progress in our laboratories.

\section{Acknowledgments}

These investigations, initiated in a cooperation between the CNRS (France) and the Hungarian Academy of Sciences (HAS) several years ago were extended to include further interested partners. Support by the OTKA Hungarian Research Fund, grants T016506, T016294, T029215, T030327, T032029, T032249 and by the HAS Aid-In-Research Fund 98-116 2.2 was essential, contribution from the bilateral Hungarian-Portuguese Scientific exchange project P-23/03 was helpful. 
The European Commission HPMF-CT-2000-01038/MCFI-2000-01879 contract is highly acknowledged. L.L. has benefited from a János Bolyai Research Fellowship, G.D., A.P.L., P.M.G., and M.F.F.M. from short-term visits within the European Commission FW5 Centre of Excellence project (contract No. ICA1-CT2000-70029).

\section{References}

[1] R. Paulin, G. Ambrosino, C.R. Acad. Sci. Paris 263, 207 (1966).

[2] For a general reference, see Principles and Applications of Positron and Positronium Chemistry, Eds. Y.C. Jean, P.E. Mallon, D.M. Schrader, World Scientific, Singapore 2003.

[3] M. Debowska, J.Ch. Abbé, G. Duplâtre, Phys. Status Solidi B 146, 91 (1988).

[4] G. Duplâtre, Zs. Kajcsos, I. Billard, L. Liszkay, K. Lázár, L. Lohonyai, H.K. Beyer, P. Caullet, J. Patarin, in: Studies in Surface Science and Catalysis 125, Eds. I. Kiricsi, G. Pál-Borbély, J.B. Nagy, H.G. Karge, Elsevier, Amsterdam 1999, p. 277.

[5] M. Eldrup, D. Lightbody, J.N. Sherwood, Chem. Phys. 63, 51 (1981).

[6] Y.C. Jean, Microchem. J. 42, 72 (1990).

[7] D.W. Gidley, W.E. Frieze, T.L. Dull, A.F. Yee, E.T. Ryan, H.M. Ho, Phys. Rev. B 60, R5157 (1999).

[8] B. Jasinska, A.L. Dawidowicz, T. Goworek, S. Radkiewicz, Phys. Chem. Chem. Phys. 2, 3269 (2000).

[9] L. Liszkay, Zs. Kajcsos, G. Duplâtre, K. Lázár, G. Pál-Borbély, H.K. Beyer, Mater. Sci. Forum 363-365, 377 (2001).

[10] Ch. Dauwe, Mater. Sci. Forum 105-110, 1857 (1992).

[11] Zs. Kajcsos, L. Liszkay, L. Varga, K. Lázár, G. Brauer, C. Dauwe, Mater. Sci. Forum 175-178, 959 (1995).

[12] G. Duplâtre, Z. Kajcsos, T. Goworek, L. Varga, L. Liszkay, I. Billard, K. Lázár, J. Radioanal. Nucl. Chem. 211, 225 (1996).

[13] Zs. Kajcsos, G. Duplâtre, L. Varga, I. Billard, L. Liszkay, L. Lohonyai, P. Caullet, J. Patarin, K. Lázár, Mater. Sci. Forum 255-257, 405 (1997).

[14] L. Liszkay, Z. Kajcsos, G. Duplâtre, K. Lázár, G. Pál-Borbély, H.K. Beyer, Mater. Sci. Forum 363-365, 377 (2001).

[15] Zs. Kajcsos, L. Liszkay, G. Duplatre, L. Lohonyai, L. Varga, K. Lázár, G. Pál-Borbély, H.K. Beyer, P. Caullet, J. Patarin, A.P. de Lima, C.L. Gil, P.M. Gordo, M.F.F. Marques, Radiat. Phys. Chem. 68, 363 (2003).

[16] J.L. Guth, H. Kessler, R. Wey, in: Proc. 7th Int. Zeol. Conf., Tokyo, Eds. Y. Murakami, A. Iijima, J.W. Ward, Kodansha-Elsevier, Tokyo 1986, p. 121.

[17] S.J. Wang, B. Wang, J. Zhu, Z. Wang, Y.Q. Dai, C.Q. He, Mater. Sci. Forum 363-365, 219 (2001).

[18] Zs. Kajcsos, G. Duplâtre, L. Liszkay, to be published in Nucl. Instrum. Methods Phys. Res. B.

[19] G. Duplatre, Zs. Kajcsos, L. Liszkay, to be published in Phys. Status Solidi A. 